\title{
Professional education of procurement transplant coordinators in Japan
}

\author{
Norihide Fukushima, Setsuko Konaka, Osamu Kato, J untaro Ashikari \\ Department of Therapeutics for End-Stage Organ Dysfunction, Osaka University, Osaka, Japan \\ Correspondence: Norihide Fukushima, MD. Address: Department of Therapeutics for End-Stage Organ Dysfunction, \\ Osaka University Graduate School of Medicine, 2 Yamada-oka, Suita, Osaka, Japan 565-0871. Telephone: \\ 81-6-6879-3294. FAX: 81-6-6879-3292. E-mail: nori@tesod.med.osaka-u.ac.jp.
}

Received: December 30, 2012

Accepted: March 6, 2013

Online Published: May 7, 2013

DOI : $10.5430 /$ jnep.v3n12p16

URL: http://dx.doi.org/10.5430/jnep.v3n12p16

\section{Abstract}

As only 81 brain dead (BD) organ donations had been performed in Japan for 13 years since 1997 because of very strict Organ Transplantation Act, the Act was revised on 17th July in 2010. By renewal of the Act, organs can be donated after BD with consent from their family, if he or she did not deny organ donation. By this renewal, there has been 4 or 5 fold increase in $\mathrm{BD}$.

There are 88 procurement transplant coordinators (PTC) in Japan. 34 PTCs belong to Japan Organ Network (JOT) and others to each administrative division. JOT has made guideline manuals of standard roles and procedures of PTC during organ procurement in BD donors and donors after cardiac death.

In order to manage increased organ donations after the revision of the Act, we needed to modify the education systems. First, the guideline manuals of PTC were modified to correspond with the revised Transplant Act and guidelines made by the Government. Second, all PTC got together in the same meeting room to learn the new organ procurement system to deal with the revised Transplant Act and guidelines. Third, the special education programs for 2 months were provided for the 5 to 10 newcomers. Last, the practical training in each donor case by elder PTC was also done for newcomers.

Topics of the education program were as follows: the revised transplant Act and guidelines, family approach for organ donation, BD diagnosis, donor evaluation and management, organ procurement and preservation, allocation system, hospital development and family care

In the future, each PTC will be divided into special categories, such as the donor family PTC, the donor management PTC and the operation room PTC. Therefore, we need to make special education program for each category in the near future.

\section{Key words}

Education, Organ procurement, Japan Organ Transplant Network, Procurement transplant coordinator, Revised Organ Transplant Act

\section{I ntroduction}

Since 1978, the donation of kidneys after cardiac death (DCD) has been legally accepted in Japan, if family consent was obtained. Small children had been able to donate their kidneys after cardiac death. The Japanese Organ Transplantation 
Act for brain dead (BD) donation was issued in October 1997. The Act required a living written consent for BD and organ donation and did not allow BD donation from children younger than 15 yrs. From these reasons, only 81 BD organ donations have been performed in Japan for 13 years since the Act was issued in October $1997^{[1]}$.

When the Japanese Organ Transplant Act was issued, Japanese Organ Transplantation Network (JOT) was established in 1997. JOT plays roles in listing recipients, management, public education and head-quarter at the time of organ donation. JOT procurement transplant coordinators (PTC) play roles in getting consent for organ donation from relatives, donor evaluation and management, organ transport, management of organ recovery and taking care of donor families during and after donation.

Every prefecture has at least one own prefectural PTC who is mainly working for public education and hospital development. They also collaborate with JOT PTC at the time of organ procurement. Most prefectures commission hospital staffs in procurement hospital to be in-hospital PTC (In-Hp PTC), who make their hospital staffs be aware of organ donation and support organ procurement (see Figure 1) ${ }^{[2,3]}$.

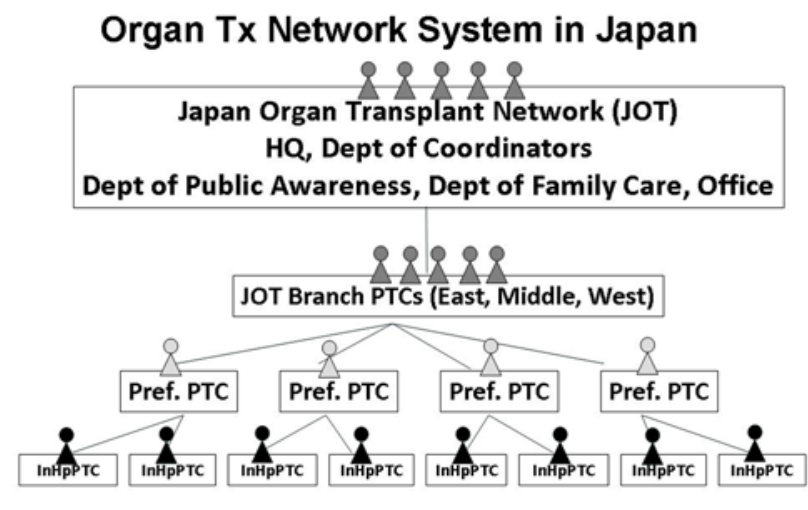

InHpPTC : In-hospital coordinator (Nurses, physicians etc.)

Figure 1. Organ transplantation network system in Japan

Finally the Act was revised on 17th July in $2010^{[1]}$. By renewal of the Act, organs can be donated after BD with consent from their family, if he or she did not deny organ donation. Although the Act was revised in 2010 and BD organ donation increased from 13 to 44 cases in a year, the number was still extremely smaller than other developed countries. The revised Act accept organ donation from BD children younger than 15 year. However, only 158 of 504 (42.4\%) procurement hospitals where BD organ donation is allowed by the Government have established organ procurement system from children. In these circumstances, In-Hp PTC may play great roles in increasing organ donation and making procurement procedure smooth.

In USA ${ }^{[4]}$, the American Board for Transplant Certification (ABTC) provides four types of certification, depending on the area of specialty: Certified Procurement Transplant Coordinator (CPTC), Certified Clinical Transplant Coordinator (CCTC), Certified Clinical Transplant Nurse (CCTN), and Certified Transplant Preservationist (CTP). In Japan, Japanese Joint Committee for Certification of Clinical Transplant Coordinators organized by Japan Transplant Society, Japan Academy of Transplantation and Regeneration Nursing, and 5 affiliate associations, started to provide certification for clinical transplant coordinators in 2011. However, there is no certification system for PTC in Japan yet.

Department of Coordinators and the coordinator committee in JOT play a main role in educating JOT-PTCs and prefectural PTCs ${ }^{[2]}$. JOT has made guideline manuals of standard roles and procedures of PTC during organ procurement in BD and DCD organ donation. Although JOT made a textbook for In-Hp PTC and has held several educational programs 
for In-Hp PTC, In-Hp PTCs have been educated mainly by prefectural PTC or their own hospital. Therefore, education system for In-Hp PTC should be modified ${ }^{[5]}$.

\section{Transplant network system in Japan}

To discuss professional education of procurement transplant coordinators (PTC) in Japan, we need to know the following situations in Japan; legislation of organ transplantation, network system for organ allocation, public awareness about organ transplantation, basic education about organ transplantation for medical students (physicians, nurses and medical engineers, etc.), and the education system for the transplant professionals.

Unlike USA ${ }^{[6]}$, European countries ${ }^{[7]}$ and Korea ${ }^{[8]}$, JOT plays a role in recipient registration and organ allocation as well as organ procurement. Therefore, JOT-PTCs need to know how to register transplant candidates in waiting list, to select a proper recipient, to send specimens to HLA laboratory, to communicate transplant centers, and to arrange transportation of procurement teams and organs.

Currently (December 2012), 88 PTCs are delegated by the Japanese Government. 34 PTCs belong to JOT and 54 are delegated by the prefecture. Although all JOT-PTCs are working in full-time base, only 33 prefectural PTCs are working in full-time base. Occupation of JOT-PTCs was nurse in 11, medical examiner in 6, medical engineer in 1, pharmacologist in 1, emergency paramedics in 1 and others in 7 . That of prefectural PTCs is nurse in 29 , medical examiner or engineer in 6, pharmacologist in 3 and others in 16. Most prefectures commission hospital staffs in procurement hospital to be in-hospital PTC (In-Hp PTC) (see Table 1). The occupation of In-Hp PTCs is nurse in 66\%, physician in 18\% and others in $16 \%$ and only $2 \%$ was a full-time In-Hp.

Table 1. Procurement transplant coordinators in Japan

\begin{tabular}{|c|c|c|c|c|}
\hline & No. & Foundation & Sphere of activity & Jobs \\
\hline JOT PTC & $34(11)^{*}$ & JOT & All Japan & $\begin{array}{l}\text { Public Awareness } \\
\text { Organ Procurement and Organ Sharing } \\
\text { (Legal Procedures-, Recipient Selection } \\
\text { Arrangement of Procurement Teams) }\end{array}$ \\
\hline Prefecture PTC & $54(35)^{*}$ & Prefecture, etc. & In Prefecture & $\begin{array}{l}\text { Public Awareness, Organ Procurement } \\
\text { Hospital Development } \\
\text { (Legal Procedures) }\end{array}$ \\
\hline In Hp PTC & $\begin{array}{l}\text { about } 1800 \\
(66 \%)^{*}\end{array}$ & $\begin{array}{l}\text { Organ } \\
\text { procurement Hp }\end{array}$ & In hospital & $\begin{array}{l}\text { Dailey Hospital Development } \\
\text { Assist Organ Procurement at donation } \\
\text { (In-Hp arrangement, Family care) }\end{array}$ \\
\hline
\end{tabular}

Note. PTC: procurement transplant coordinators, JOT: Japan Organ Transplant Network, ( )*: nurse in PTCs

\subsection{Japan Organ Transplant Network}

When the Japanese Organ Transplant Act was issued, JOT was established in 1997. Only JOT has had a license for organ mediation in Japan since the former Transplant Act was issued in 1997. JOT plays a role in listing recipients, management, public education and head-quarter at the time of organ donation. JOT-PTCs play roles in getting consent for organ donation from relatives, donor evaluation and management, organ transport, management of organ recovery and taking care of donor families during and after donation as well as hospital development.

As only JOT has had a license for organ mediation in Japan, JOT-PTCs can mediate organs from BD and DCD donors to transplant candidates. Prefectural PTCs are delegated by JOT to mediate organs in collaboration with JOT-PTCs. 


\section{J OT headquarter}

The office of JOT headquarter (HQ) has been located in Tokyo. The HQ plays roles in listing organ transplant candidates, organ allocation and organ procurement management during BD organ donation, public relations, organ donationtransplant registries and care of donor family.

Department of Coordinators and the coordinator committee in JOT play roles in arranging daily activities and organ procurement of PTCs and in educating JOT-PTC and prefectural PTCs.

\section{JOT regional branches}

Three regional branches (East, Middle and West JOT branches) have been located in Tokyo, Nagoya and Osaka. These branches play roles in kidney allocation at DCD donation, organ allocation and organ procurement management during $\mathrm{BD}$ and DCD organ donation, care of donor family in their region, hospital development and public relations in collaboration with prefectural PTCs.

\subsection{Prefectural procurement transplant coordinators (PTCs)}

Every prefecture has at least one own prefectural PTC who is mainly working for public education and hospital development. They also collaborate with JOT-PTCs at the time of organ procurement preformed in neighbor prefectures as well as their own prefecture.

To delegate to mediate organs to prefectural PTCs, prefectural PTCs need to attend annual or interim seminar and pass an interview and written examination. As there are large variations in occupation, career, education and experiences of organ procurement among prefectural PTCs, prefectural PTCs are divided into two groups; class-1 (basic prefectural PTC) and class-2 (advanced prefectural PTC).

\subsection{I n hospital procurement transplant coordinators (I n-Hp PTCs)}

Although DCD kidney procurement is allowed in any hospital in Japan by the Transplant Act and guideline made by Japanese Guideline since 1978, only about 500 hospitals are allowed to undergo BD organ procurement, Moreover, only 158 of these procurement hospitals have established organ procurement system from children.

Most prefectures commission hospital staffs in procurement hospital to be In-Hp PTC. In 40 prefectures, In-Hp PTC is delegated by the prefectural Government and kidney and/or eye bank etc. But, in 4 prefectures (Tokyo, Chiba, Saitama and Osaka), no In Hp PTC is delegated. Only $2 \%$ of In-Hp PTC is working in full-time base ${ }^{[5]}$.

In-Hp PTCs mainly play a role in making their own manual for organ procurement, awareness of organ donation to patients and medical staffs, arranging the in-hospital transplant seminars, consultation about organ transplantation and donation, organizing simulation of organ donation, as well as coordinating the donation case. At organ donation, they coordinate in-hospital staffs and arrange organ procurement, communicate with prefectural and JOT-PTC, take care of donor family and take informed consent for organ donation in collaboration with prefectural or JOT-PTC.

To increase organ donation, the recognition of importance of organ transplantation and donation by medical staffs in donor hospitals is very important. Frequent visits to donor hospital by JOT-PTCs or prefectural PTCs are very hard to do, but are most effective method for hospital development. By frequent visits, PTCs can find a key person for organ donation in each donor hospital and make good relationship with medical staffs in the hospital. Seminars for the In-Hp PTCs and simulation of organ donation in each hospital are also effective. Moreover, seminars for medical staffs performing BD determination and medical staffs evaluating donors and managing donors until and during the organ procurement surgery were important for hospital development. 


\section{Roles of J OT-PTCs and prefectural PTCs at time of organ donation}

Time course of BD organ donation is summarized in Table 2. At time of organ donations, 5 to 8 JOT-PTCs or prefectural PTCs share their duties (see Figure 2) (family consent and care, donor assessment and management, coordination of procurement operation, and transportation of procurement team and organs) ${ }^{[9]}$ in collaboration with In-Hp PTCs delegated in the hospital.

Table 2. Time course of brain dead (BD) organ donation

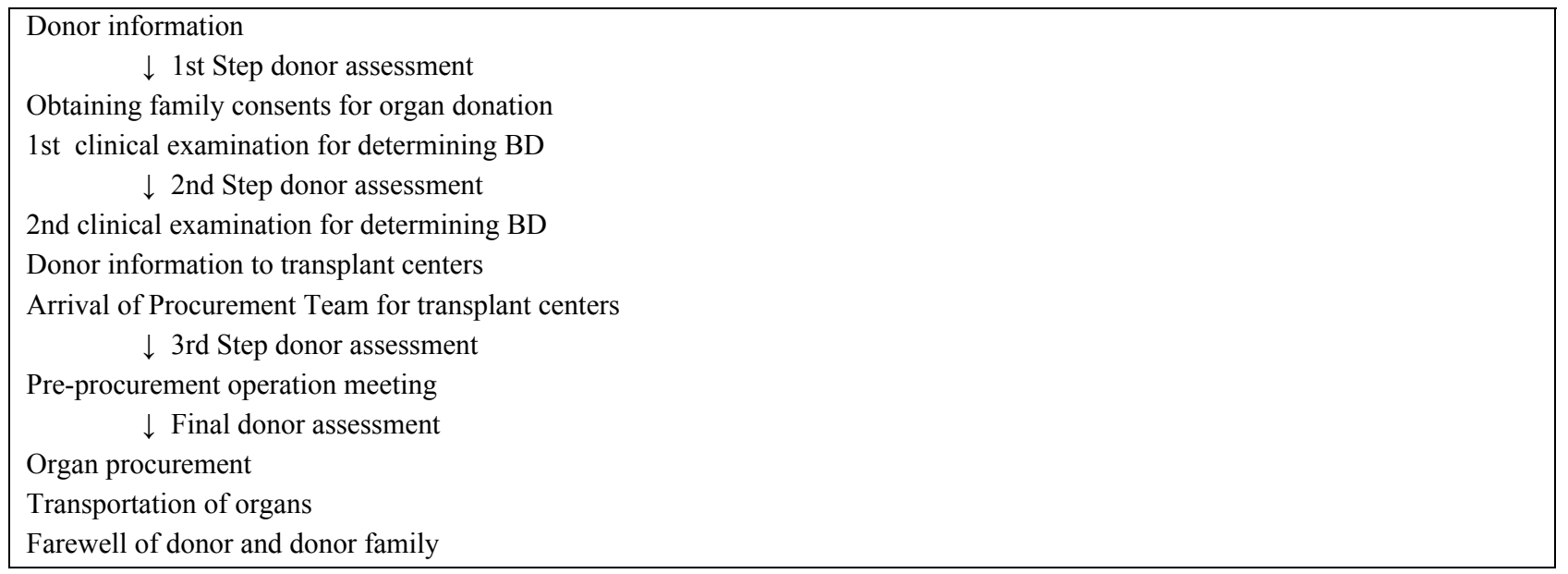

\section{Communication Network among PTC} in brain dead organ procurement

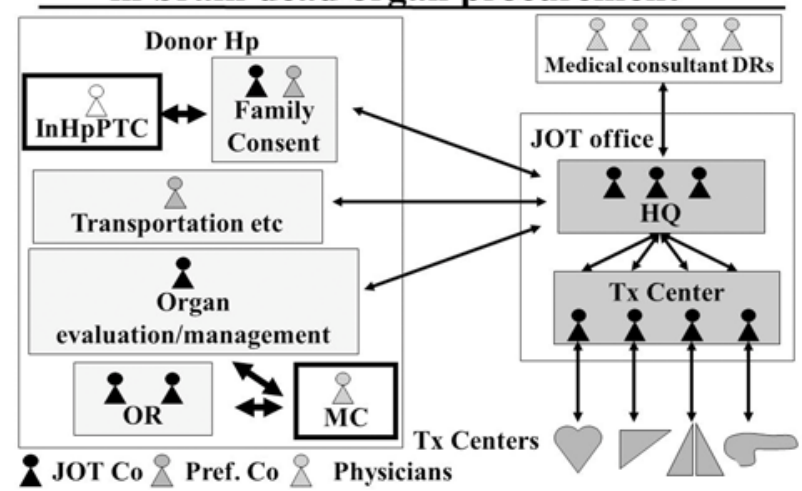

Figure 2. Communication network among procurement transplant coordinators in brain dead organ procurement

\subsection{Family approach and informed consent}

The PTC ensures that the legal next-of-kin (NOK) is provided the option of organ donation in a sensitive and caring manner that meets his/her emotional and cultural needs.

Soon after arriving at procurement hospital, PTCs communicates with the physician and hospital staff to learn of the patient's clinical status and family's understanding of the clinical condition and to ensure the family understands the hopelessness of the patient's clinical condition prior to the discussion of donation. After providing the family with an overview of their options for donation, addressing the family's questions, concerns, and needs, supporting the family regarding the donation decision, utilizing donor registry status, and ensuring the family understands the need for all 
evaluation procedures, which organs and tissue will be recovered, and the timing of the entire donation process, PTCs explain the consent forms, obtain informed consent and ensure written documentation of the in-person, telephone, or translated consent in the patient's medical record as well as the donor record.

Because organ donation from BD donors has become possible with consent from the family, the psychological burden on the donor's family has increased. Therefore, while maintaining the quality of PTCs who can properly understand the intention of the donor's family, the number of PTCs should be increased to cope with the future rise in organ donation. Some transplant physicians praise a "good" PTCs for achieving a high rate of organ donation, but it is more important for a PTC to properly understand the thoughts and feelings of the donor's family on site even if organ donation is ultimately impossible.

In Japan, family members are usually staying in the hospital until the procurement operation is finished. Therefore, PTCs should take care of family members throughout PTCs also discuss with the families about a plan for follow-up contact with the family, before and after the surgical organ and tissue recovery.

\subsection{Current organ donor assessment system in Japan}

As donor shortage is extremely severer in Japan than other developed countries due to very strict Transplant Act, JOT has established original donor assessment and management system in Japan. Since 2002, medical consultant transplant surgeons (MCs) were sent to the hospital ${ }^{[9]}$. Current organ and recipient transplanted from one donor is 5.5 organs and 5 recipients in Japan, respectively.

\section{1st step donor assessment}

JOT-PTCs are called to a donor hospital if there is a potential BD donor. They listen to patient clinical course from medical staffs and check clinical records in order to find out whether the patient is suitable for organ donation. If there are no absolute contraindications for organ donation, such as untreated malignancy and several viral infections, they get informed consent for BD organ donation from his or her family. Then legal examination for BD is carried out.

\section{2nd step donor assessment}

After completion of 1 st clinical examination for determining BD, MCs are sent to the hospital ${ }^{[4]}$. They and JOT-PTCs check clinical records such as clinical course before and after BD, medication given, blood examination, electrocardiogram, chest X-ray and abdominal and chest computed tomography (CT) scan and undergo ultrasound examination for heart, liver, pancreas and kidneys and bronchofiberscopy (BFS) by themselves. MCs also rule out malignancies from findings of CT scan and ultrasound examination. JOT-PTCs make donor evaluation sheets which will be sent to transplant centers later.

After 2nd clinical examination for determining BD is completed and the patient is sentenced death, donor information such as donor evaluation sheets and images of ECG, chest-X ray, ultrasound examinations, BFS and CT scans is sent to transplant centers using a mobile system. Then transplant centers decide whether their recipient undergo transplant from that $\mathrm{BD}$ donor and their procurement team is sent to the hospital

\section{3rd step donor assessment}

After arriving at the donor hospital, their procurement team also evaluate the condition of donor organs by ultrasound examinations for the heart and abdominal organs and BFS by themselves in the intensive care unit (ICU), before procurement operation ${ }^{[9]}$. They assess organ function and determine whether the organ can be transplanted to their recipient. 


\section{Final donor assessment}

After opening the chest and abdomen, the procurement team evaluates organs by watching and touching carefully. Usually liver biopsy is performed to rule out more than moderate grade fatty liver and malignancies. They also rule out unexpected malignancies in the pleural and abdominal cavities.

\subsection{Pre-procurement meeting and management of procurement operation}

Before starting procurement operation, all procurement surgeons, anesthesiologists and OR nurses gather in the meeting room. They negotiate on the types of procured organs, how to procure each organ (e.g. organ dissection/perfusion technique, incision lines, blood drainage technique, et al), what kinds of samples (e.g. blood, lymph nodes and spleen) are needed, and how to manage the donor during operation. JOT-PTCs arrange and manage the meeting ${ }^{[9]}$.

As most Japanese anesthesiologists never have an experience of management of procurement operation from BD donors, MC also supports them to stabilize the donor hemodynamics during operation. Skillful staff surgeons, not resident surgeons, harvest their donor organ.

As an inverse relationship between volume of intraoperative colloid and early lung allograft function is reported, packed red blood cells and albumin are transfused to maintain circulating blood volume and to replace proteins and fluids during procurement operation. To improve organ perfusion with preservation solution, any catecholamine is additionally administered as less as possible to dilate the vessels of organs and antidiuretic hormone (ADH) infusion and all catecholamine infusion are discontinued at the time of bolus infusion of heparin sulfate $(400 \mathrm{U} / \mathrm{Kg})$.

\subsection{Donor family care}

Because the donor's family members live long after the loss of their loved one, it is necessary to exert maximal effort to keep them content after donation. Conventionally, it has been the general practice that JOT-PTC or prefectural PTCs involved in acquisition of consent visit the family periodically and thank them by placing a letter of appreciation in their hands, but this is far from adequate. In addition, the donor's family members may suffer posttraumatic stress disorder due to the bereavement, and a system that provides informal counseling exclusive to JOT-PTCs at any time should therefore be formulated. It is also important to establish a framework for cooperation with psychologists and psychiatrists.

\section{Education of PTCs}

\subsection{Education of the JOT-PTCs}

\section{Education of the rookie J OT-PTCs}

For the rookie JOT PTCs, the special education programs for 2 months were provided (see Table 3) ${ }^{[2,3]}$. The topics of the classroom lectures were the overview of transplant medicine, the previous and revised organ transplant Act, the history of organ transplantation in Japan and in the world, what was JOT and organ transplant network system, the roles and tasks of PTC in organ donation (BD and DCD), the current status and problems in organ transplantation (heart, lung, liver, pancreas and kidney), the therapies for end-stage organ failure, the process and roles of PTCs in BD organ donation under the revised Act, donor evaluation and management, the family consent at BD donation and DCD.

After the 2-month classroom lectures, they were assigned to the JOT branch and the on-job training was done by elder JOT-PTCs including their preceptor or the chief of branch JOT-PTC. They also attend several courses of 1 or 2-day seminar (Table 3) held at JOT-HQ office to learn more practical procedure than the orienteering education program. At 
that seminar, they present their favorite topic such as family care, donor assessment or hospital development to get skills to summarize and present the topics.

Table 3. Education for rookie Japan Organ Transplant Network procurement transplant coordinators

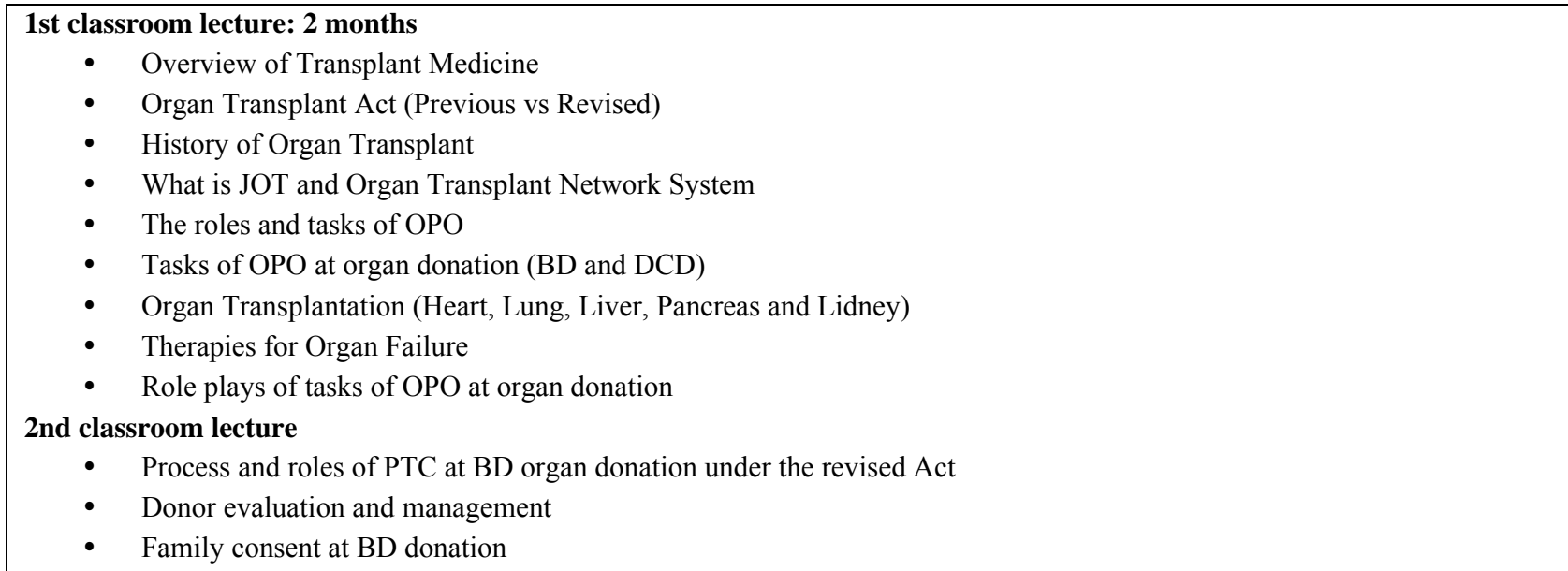

Note. OPO: organ procurement organization, BD: brain dead organ donation, DCD: donation after cardiac death

PTC competency self-assessment checklist is given to each JOT-PTC and they put the self-assessment of each task on the checklist 1, 3, 6, 9 and 12 months after entering JOT. At the same time, the supervisor interviews them to confirm their skills. There are 3 steps to approve their skills: reading and understanding, performing procedure under supervision and by their selves. All records are witnessed or approved by their preceptors or supervisors.

\section{Education of the elder J OT-PTCs}

For the elder JOT PTCs (Table 4), details of BD organ donation under the revised Act, details of donor assessment and management before and during procurement operation, details of pediatric organ donation, such as pediatric emergency therapy, care of pediatric family, and organ procurement in children were taught. Simulations of family consent in special condition, such as, how to deny any refusal of the person for organ donation, how to deny child abuse, how to confirm consent of pediatric family and how to confirm consent of priority organ donation to relatives were also performed. MCs provide the on-job-training of donor assessment and management before and during procurement operation.

Table 4. Education for already hired Japan Organ Transplant Network procurement transplant coordinators

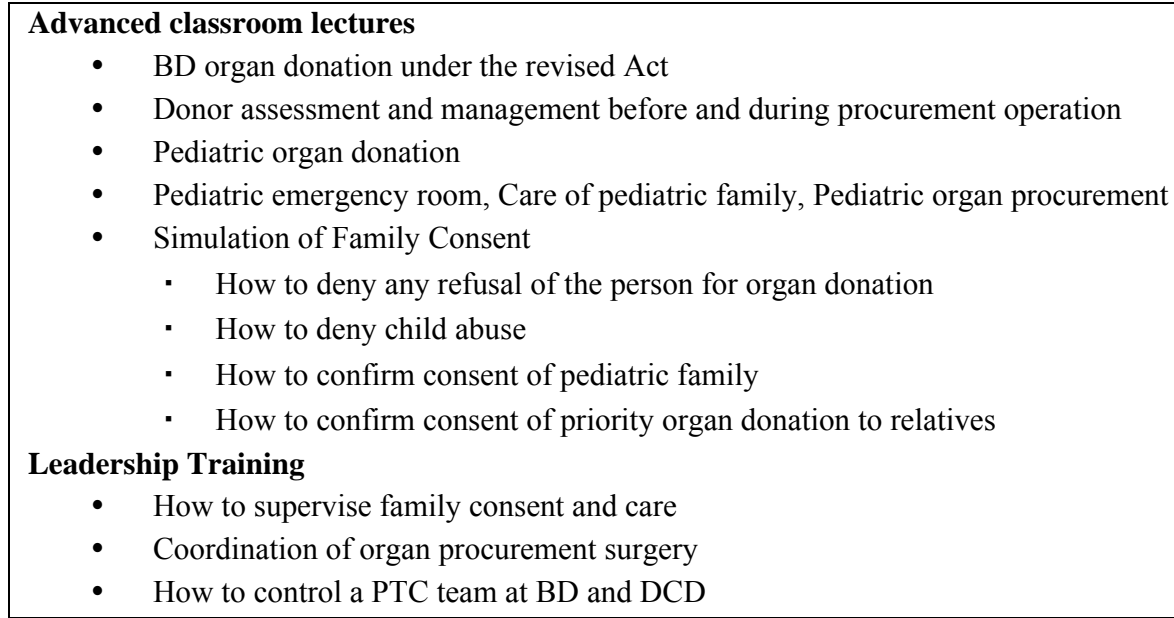

Note. BD: brain dead organ donation, DCD: donation after cardiac death 


\section{Education of the leader J OT-PTCs}

For the leader JOT-PTCs, how to supervise family consent and care, to assess and manage organ donors, to coordinate organ procurement surgery and to control and PTC team in BD and DCD donation are trained.

\section{Working groups for education of J OT-PTC and prefectural PTCs}

In 2011, four working groups (WGs) for education of JOT-PTCs and prefectural PTCs are established in JOT: Organ allocation, family approach, donor assessment and management and procurement surgery. 3 to 4 JOT-PTCs consist of each WG and are currently making education program and tools. These education programs will be started in April 2014.

\subsection{Education for the prefectural PTCs}

To delegate to mediate organs to prefectural PTCs, every prefectural PTCs need to attend annual or interim seminar and pass an interview and written examination. Class-1 (basic) and class-2 (advanced) prefectural PTCs needs to have different educational programs.

Annual 3-day seminar for the prefectural PTCs has been held in every February. The topics of the program are the process of DCD and BD organ donation (initial action, family consent, donor evaluation etc.) and the roles of PTC, especially the prefectural PTC in DCD and BD donation, standard process of priority organ donation to relatives, case studies (DCD and BD donation), information disclosure to Media, family consent at BD donation. Class-1 PTCs attend all programs and class-2 attend 2nd and 3rd day program. To delegate class-1 and -2 PTCs, they need to been interviewed by the members of JOT coordinator committee.

Each JOT branch has conducted its own classroom lectures 3-5 times a year (see Table 5). The topics were current status of DCD and BD donation in each branch or special prefecture with case studies, how to proceed pediatric organ donation, family care during and after donation, case studies (DCD and BD donation) and public awareness and hospital development.

Table 5. Education for prefectural procurement transplant coordinators (PTCs)

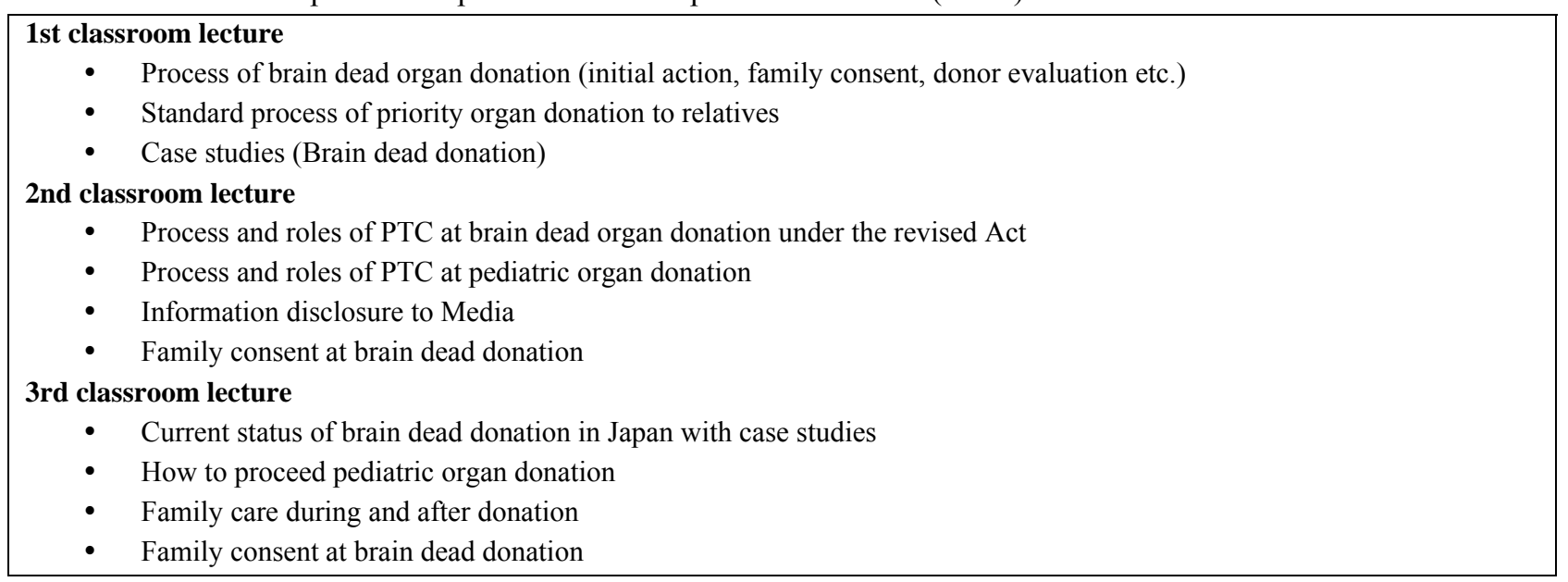

\subsection{Education for the in-hospital PTCs}

JOT-PTCs and prefectural PTCs conducted classroom lectures 1-3 times a year. The topics were legislation of organ transplantation/donation in Japan, current status of DCD and BD organ donation in Japan, the process of DCD and BD organ donation (initial action, family consent, donor evaluation etc.), the roles of In-Hp PTCs in DCD and BD organ donation, case studies (DCD and BD donation), information disclosure to Media and family consent at BD donation. 
A simulation with classroom lectures of DCD or BD donation in each hospital was effective to education of the In-Hp PTCs as well as hospital development. In order to perform the simulation, In-Hp PTCs and medical staffs needed to establish their own organ donation system for each hospital, to make their own guideline and manual of organ donation and to figure out the roles of each hospital staffs in organ donation.

In order to establish education system for In-Hp PTCs, we underwent national survey using 28-item self-designed questionnaire that queried ${ }^{[5]}$; 1 . occupation and status, 2. activities and issues (daily and at organ donation), 3. education and experiences of organ donation. 4 . what he or she wants to learn about organ donation. $52 \%$ of respondents worked in organ procurement hospitals where have been allowed to undergo BD organ donation. 38\% worked in those where have been allowed to undergo only DCD donation. Only $46 \%$ worked in donor hospitals where cadaver organ donations were undergone in past. The occupation of the respondents was nurse in $66 \%$, physician in $18 \%$ and others in $16 \%$ and only $2 \%$ was a full-time In-Hp PTC.

However, it was very difficult for them to do their daily activities, to manage a rare and sudden donation case and to find time to learn organ donation, because they had another post. Although $77 \%$ have attended any seminars about organ donation projected by JOT, the prefecture PTC, academic associations for organ or tissue transplantation and Japan Transplant Coordinator Organization (JATCO), 93\% still wanted to take more professional education. Nurses and medical social worker were more likely to desire to take more professional education than physicians and medical examiners.

The topics which they desired to learn were donor family care, overall procedures of organ/tissue donation, the role of In-Hp PTC, how to project simulation of organ donation, the legislation and social system of organ donation, medical indications of organ donation, the current status of organ donation and transplantation in Japan, donor assessement and management and case studies. There were significant variations in interested topics among their occupations.

From the result of this national survey, our department held special educational seminar for In-Hp PTC to establish educational program for In-Hp PTC in Japan (2 lectures/day for 10 days) from May to October, 2012. Topics of this seminar were history of transplantation, current status of organ transplantation and donation in Japan, legislation and network system of organ transplantation in Japan, outcomes of organ transplantation in Japan, nursing care of transplant recipients, pathophysiology and diagnosis of $\mathrm{BD}$, the role of PTC, process of organ donation, sight visit of JOT office, family care and informed consent for organ donation, survey of donor family, donor evaluation and management, role of In-Hp PTCs, case studies and simulation of organ donation, pediatric organ donation, determination of child abuse, informing organ donation, and care of family of end-life patients. By survey of 16 participants, all of them were satisfied to the program. But they still wanted to have on-job training.

Although we may be able to establish education curriculum for In-Hp PTC in the near future, national support is essential to provide these programs to all In-Hp PTC in Japan.

\section{Discussion}

Although the Transplantation Act was revised in 2010 and BD organ donation increased from 13 to 44 cases in a year, the number was still extremely smaller than other developed countries. In these circumstances, In-Hp PTC may play great roles in increasing organ donation and making procurement procedure smooth.

Currently, JOT PTCs and prefectural PTCs have conducted a classroom lecture for In-Hp PTC and other medical staffs in each donor hospital. The topics are legislation of organ transplantation/donation in Japan, current status of DCD and BD organ donation in Japan, the process of DCD and BD organ donation (initial action, family consent, donor evaluation etc.), the roles of medical staffs at DCD and BD organ donation. Indeed, $77 \%$ of the respondents have attended these seminars. 
However, 93\% still wanted to take more professional education. Nurses and medical social worker were more likely to desire to take more professional education than physicians and medical examiners ${ }^{[5]}$.

As most of In-Hp PTCs have another post, they can find only a short period (1 or 2 days) to take the professional education, such as lectures. Even at organ donation, they cannot preferentially involve in organ donation and feel sorry to their partners because they cannot do their daily jobs. Therefore, it is very difficult for them to find time to take practical on-job training ${ }^{[5]}$.

Of course, it might be effective to establish full time In-Hp PTC in every donor hospital. However, it is very hard to make such a post in every hospital, because the number of organ donation has been extremely smaller in Japan than other developed countries. Therefore, we need to establish special educational program for In-Hp PTC in Japan.

In the future, each JOT-PTC will be divided into special categories, such as the donor family PTC to obtain informed consent for organ donation and care donor families until the donor leaves donor hospital, the donor management PTC to evaluate donor organs and manage the donor until procurement surgery and the operation room PTC to manage organ procurement surgery. Therefore, we need to make special education program for each category in the near future.

\section{References}

[1] Fukushima N. Revised Organ Transplant Act and Transplant Surgeons. Jpn Med Assoc J. 2011; 54(6): 387-391

[2] Fukushima N, Konaka S, Kato O, Ashikari J. Professional education and hospital development for organ donation. Transplant Proc. 2012; 44(4): 848-50. PMid:22564564 http://dx.doi.org/10.1016/j.transproceed.2012.03.049

[3] Konaka S, Kato O, Ashikari J, Fukushima F. Modification of education system for organ procurement coordinators in Japan after the revision of the Japanese Organ Transplantation Act. Transplant Proc. 2012; 44(4): 851-4. PMid:22564565 http://dx.doi.org/10.1016/j.transproceed.2012.03.012

[4] The American Board for Transplant Certification (ABTC) Certification for Clinical and Procurement Transplant Clinicians. Available from: http://www.natcol.org/Professional-Development/ ABTC.asp

[5] Konaka S, Kato O, Ashikari J, Fukushima F. Current status of in-hospital donation coordinators: Nationwide survey in Japan. Transplant Proc 2013 (in press)

[6] Sung RS, Galloway J, Tuttle-Newhall JE, Mone T, et al. Organ donation and utilization in the United States, 1997-2006. Am J Transplant. 2008; 8(4 Pt 2): 922-34. PMid:18336696 http://dx.doi.org/10.1111/j.1600-6143.2008.02171.x

[7] Langer RM, Cohen B, Rahmel A. History of eurotransplant (ET) Transplant Proc. 2012 Sep; 44(7): 2130-1. PMid:22974933 http://dx.doi.org/10.1016/j.transproceed.2012.07.125

[8] Korean Network for Organ Sharing: Organ transplantation statistics. Available from: http://konos.go.kr.

[9] Fukushima N, Ono M, Nakatani T, et al. Transplant Proc. Strategies for maximizing heart and lung transplantation opportunities in Japan. 2009; 41(1): 273-6. 